\title{
Kabuki Syndrome
}

National Cancer Institute

\section{Source}

National Cancer Institute. Kabuki Syndrome. NCI Thesaurus. Code C124837.

A rare, autosomal dominant or X-linked dominant inherited syndrome caused by mutations in the KMT2D gene (also known as MLL2) or the KDM6A gene. It is characterized by distinctive facial features including arched eyebrows, long eyelashes, long palpebral fissures with the lower lids turned out at the outside edges, a flat nose, and large protruding earlobes, developmental delay and intellectual disability. 